Copyright 2000 American Association of Physics Teachers (AIP Publishing). This article may be downloaded for personal use only. Any other use requires prior permission of the author and AIP Publishing.

The following article appeared as: Penner, A.R. (2000). Suspension of a disk on a surface of water. American Journal of Physics, 68(6), 549-551, and may be found at http://dx.doi.org/10.1119/1.19495 


\title{
Suspension of a disk on a surface of water
}

\author{
A. Raymond Penner \\ Physics Department, Malaspina University-College, Nanaimo, British Columbia, Canada
}

(Received 13 May 1999; accepted 3 September 1999)

The suspension of a disk on the surface of water by the forces of surface tension and buoyancy is considered. Both theoretical and experimental results are presented along with a discussion of the relative importance of the buoyant force. (C) 2000 American Association of Physics Teachers.

\section{INTRODUCTION}

The suspension of an object by the force of surface tension is a topic discussed briefly in many introductory physics textbooks. Typically the accompanying diagram and description make it appear that the liquid surface behaves as an elastic membrane holding the object up completely by the force of surface tension. Though this imagery may be useful it is incorrect in that any real object will break through the liquid surface and the resulting upward force will, in general, involve both the surface tension and the buoyant force. The suspension of particles at fluid interfaces has previously been considered by Rapacchietta. ${ }^{1,2}$ This was a theoretical investigation of horizontal cylinders and spheres being supported by a liquid and determined the maximum size of such particles able to achieve equilibrium. The analysis did not specifically consider the relative contribution of the buoyant force to the total upward force.

This paper will consider the specific phenomenon of a disk being suspended on the surface of water. A disk was chosen as it both simplifies the theoretical analysis and lends itself to experimental verification. The equation of the resulting water surface profile will be derived, and the theoretical depth a disk will sink will be compared with experimental measurements which were carried out on two different disks. Also a theoretical determination of the relative importance of the buoyant force for various disk sizes and densities will be presented.

\section{THEORY}

The equation governing the shape of the liquid-air interface while supporting a disk can be determined by considering the equilibrium state of a horizontal section of the liquid. A similar analysis was undertaken by Rapacchietta and in several papers which considered the shape of a sensile drop. ${ }^{3,4}$ Referring to Fig. 1, the three vertical forces acting on a given horizontal section of the liquid are $F_{g}$, the gravitational force, $F_{p}$, the net force due to the pressure differential on the two faces of the given section and $F_{s}$, the net vertical component of the surface tension. In terms of the parameters indicated in Fig. 1 these forces are

$$
\begin{aligned}
F_{g}= & -\rho g \pi\left(R^{2}-x^{2}\right) \Delta y, \\
F_{p}= & \rho g\left(y_{s}-y\right) \pi\left(R^{2}-x^{2}\right) \\
& -\rho g\left(y_{s}-(y+\Delta y)\right) \pi\left(R^{2}-(x+\Delta x)^{2}\right), \\
F_{s}= & 2 \pi \gamma(x+\Delta x) \sin (\theta+\Delta \theta)-2 \pi \gamma x \sin \theta,
\end{aligned}
$$

where $\gamma$ is the surface tension and $\rho$ is the density of the liquid. The vertical displacement is measured from the top of the suspended disk and $y_{s}$ represents the depth the top of the disk is below the liquid surface. The equilibrium condition for the horizontal section is

$$
F_{s}+F_{p}+F_{g}=0
$$

which reduces to

$$
\begin{aligned}
\rho g\left(y_{s}-y\right) x & =-\gamma(x \cos \theta \Delta \theta / \Delta x+\sin \theta) \\
& =-\gamma \Delta(x \sin \theta) / \Delta x .
\end{aligned}
$$

Using $y^{\prime}=d y / d x=\tan \theta$ allows for the substitution $\sin \theta$ $=y^{\prime} /\left(1+y^{\prime 2}\right)^{1 / 2}$ in Eq. (2c) and simplification leads to the following equation for the surface profile,

$$
y^{\prime \prime}=-y^{\prime}\left(1+y^{\prime 2}\right) / x-(\rho g / \gamma)\left(y_{s}-y\right)\left(1+y^{\prime 2}\right)^{3 / 2} .
$$

This second-order differential equation was solved numerically using the following Runge-Kutta-Nystrom method. ${ }^{5}$ Given the values $x_{n}, y_{n}, y_{n}^{\prime}$ from the $n$th step along the profile and the incremental value $h$, in the $(n+1)$ th step of the method the following auxiliary quantities are computed:

$$
\begin{aligned}
& A_{n}=\frac{1}{2} h y^{\prime \prime}\left(x_{n}, y_{n}, y_{n}^{\prime}\right), \\
& B_{n}=\frac{1}{2} h y^{\prime \prime}\left(x_{n}+1 / 2 h, y_{n}+\beta_{n}, y_{n}^{\prime}+A_{n}\right),
\end{aligned}
$$

where $\beta_{n}=1 / 2 h\left(y_{n}^{\prime}+1 / 2 A_{n}\right)$,

$$
\begin{aligned}
& C_{n}=\frac{1}{2} h y^{\prime \prime}\left(x_{n}+1 / 2 h, y_{n}+\beta_{n}, y_{n}^{\prime}+B_{n}\right), \\
& D_{n}=\frac{1}{2} h y^{\prime \prime}\left(x_{n}+h, y_{n}+\delta_{n}, y_{n}^{\prime}+C_{n}\right),
\end{aligned}
$$

where $\delta_{n}=h\left(y_{n}^{\prime}+C_{n}\right)$.

The values for the next step along the profile are then given by

$$
\begin{aligned}
& x_{n+1}=x_{n}+h, \\
& y_{n+1}=y_{n}+h\left(y_{n}^{\prime}+K_{n}\right),
\end{aligned}
$$

where $K_{n}=1 / 3\left(A_{n}+B_{n}+C_{n}\right)$, which is an approximation for $y\left(x_{n+1}\right)$ and

$$
y_{n+1}^{\prime}=y_{n}^{\prime}+J_{n},
$$

where $J_{n}=1 / 3\left(A_{n}+2 B_{n}+2 C_{n}+D_{n}\right)$, which is an approximation for $y^{\prime}\left(x_{n+1}\right)$. The incremental value, $h$, was set at values ranging from 0.0001 to $0.00001 \mathrm{~mm}$ and trial values of $y_{s}$ were used until a self-consistent solution, namely $y(x$ $\rightarrow \infty)=y_{s}$, was obtained.

The initial position values are $x_{1}$, the radius of the suspended disk, and $y_{1}=0$. The initial first derivative value $y_{1}^{\prime}$ is determined by the equilibrium condition for the disk. As Fig. 1 shows, the disk breaks through the surface and both surface tension and the buoyant force will contribute to the upward force. The benefit of analyzing a disk as compared to a sphere or cylinder is that the contact length is independent 


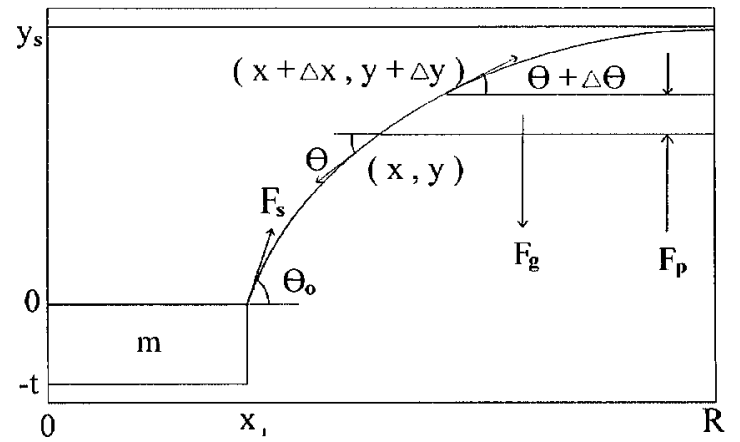

Fig. 1. Forces acting on a horizontal section of the supporting liquid. $R$ is the outer diameter of the resulting ring section and $\theta$ is the angle the tangent to the surface makes with the horizontal. The mass, radius, and thickness of the supported disk are $m, x_{1}$, and $t$, respectively.

of $\theta_{0}$, the angle the liquid subtends with the horizontal at the point of contact with the disk. This is because as the mass or density of the disk is increased the liquid will continue to adhere to the top edge of the disk. The resulting equilibrium condition for a disk suspended on a liquid surface is therefore

$$
\rho g\left(y_{s}+t\right) \pi x_{1}^{2}+\gamma 2 \pi x_{1} \sin \theta_{0}=m g
$$

and the initial value

$$
y_{1}^{\prime}=\tan \theta_{0}=\tan \left(\sin ^{-1}\left(\left(m g-\rho g\left(y_{s}+t\right) \pi x_{1}^{2}\right) / 2 \pi x_{1} \gamma\right)\right) .
$$

Figure 2 shows the theoretical surface profile generated using Eqs. (3)-(7) for a disk of radius $10.00 \mathrm{~mm}$ and thickness $1.00 \mathrm{~mm}$ and various densities. The variation in densities is equivalent to adding mass to the top of the disk. As is shown in Fig. 2, as the density of the disk is increased it will sink further below the surface leading to an increase in the upward force of surface tension, through the change in the angle $\theta_{0}$. The upward force due to the pressure exerted by the water on the bottom face also increases. It is interesting to note that Archimedes' principle is not applicable in this problem as the buoyant force depends solely on the depth the bottom face is below the equilibrium surface position. The critical case for the disk occurs when $\theta_{0}$ is equal to $\theta_{c}$, the contact angle between the disk and water. Adding further mass or increasing the density of the disk will result in the water creeping inward on the top surface which will reduce

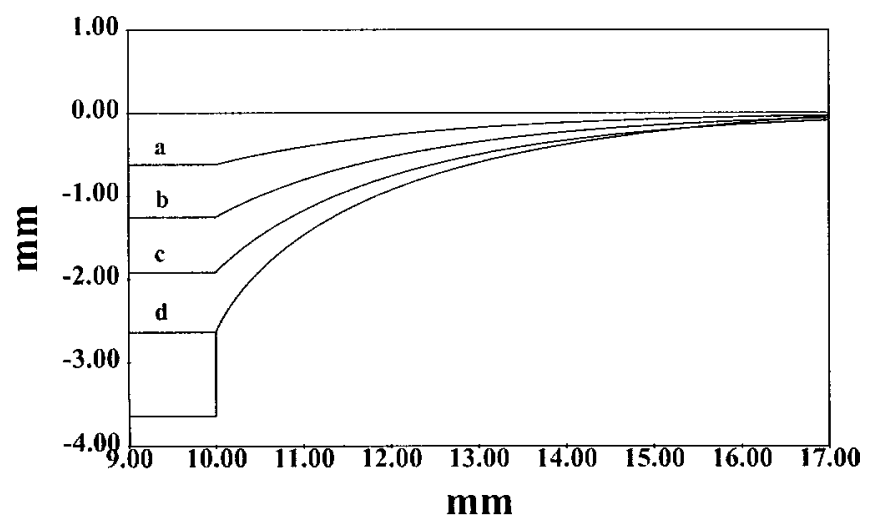

Fig. 2. Surface profiles for disk densities of (a) $2.00 \mathrm{~g} / \mathrm{cm}^{3}$, (b) $3.00 \mathrm{~g} / \mathrm{cm}^{3}$, (c) $4.00 \mathrm{~g} / \mathrm{cm}^{3}$, (d) $5.00 \mathrm{~g} / \mathrm{cm}^{3}$.

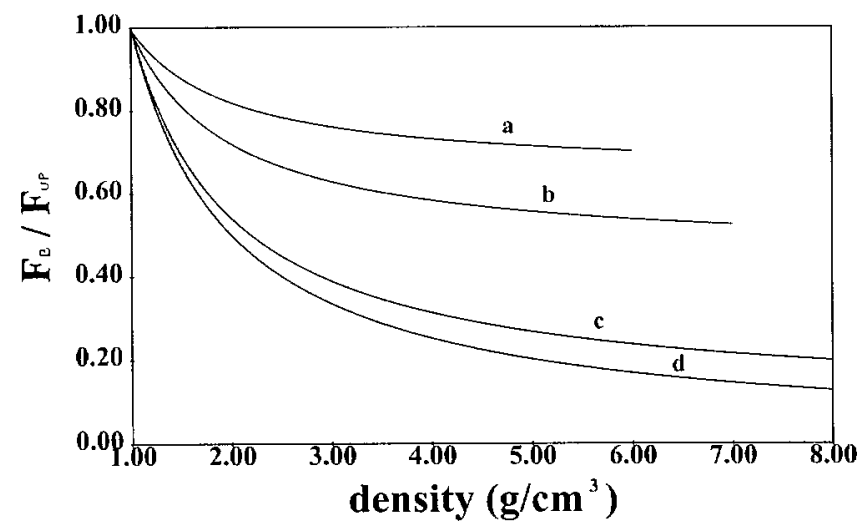

Fig. 3. Relative contribution of buoyant force for a disk of (a) thickness $=1.00 \mathrm{~mm}$ and radius $=10.00 \mathrm{~mm}$, (b) thickness $=1.00 \mathrm{~mm}$ and radius $=5.00 \mathrm{~mm}$, (c) thickness $=1.00 \mathrm{~mm}$ and radius $=1.00 \mathrm{~mm}$, (d) thickness $=0.10 \mathrm{~mm}$ and radius $=0.10 \mathrm{~mm}$.

the contact length and the disk will no longer be supported by the water surface. For disk materials where $\theta_{c}>90^{\circ}$ the critical point will occur at $\theta_{0}=90^{\circ}$ as the upward vertical force will decrease for $\theta_{0}>90^{\circ}$ and the disk will be unstable.

The relative importance of the buoyant force was considered for several different disk sizes. These were a $0.1-\mathrm{mm}$ thick disk of $0.1-\mathrm{mm}$ radius, a 1 -mm-thick disk of $1-\mathrm{mm}$ radius, a 1-mm-thick disk of 5-mm radius and a 1-mm-thick disk of $10-\mathrm{mm}$ radius. The results are shown in Fig. 3. As indicated, the relative contribution of the buoyant force decreases with increasing density. Also as indicated, the importance of the buoyant force increases with the radius of the disk. This is expected as the buoyant force is proportional to $r,{ }^{2}$ the area of the bottom face, while the force being provided by the surface tension is proportional to $r$, the circumference of the top face.

\section{EXPERIMENTAL RESULTS}

In order to confirm the above-mentioned theoretical surface profile, Eq. (3), measurements were made of $y_{s}$, the vertical displacement of the surface, versus the equivalent density of two different disks. This was accomplished by use of a traveling microscope which measured both the vertical position of the top surface of the disk and the vertical position of the water surface several centimeters from the disk. Ten independent measurements were made for each additional mass added to the disks leading to an uncertainty in the vertical displacement of $\pm 0.04 \mathrm{~mm}$.

Figure 4 shows the results for an acrylic disk of radius $(12.70 \pm 0.02) \mathrm{mm}$ and thickness $(1.78 \pm 0.02) \mathrm{mm}$ on the surface of the water. Measurements were made for every 100 $\mathrm{mg}$ of mass added to the top surface of the disk which is equivalent to a change in the density of $0.111 \mathrm{~g} / \mathrm{cm}^{3}$. The disk broke through the surface at a total mass of $2.12 \mathrm{~g}$ or equivalent density of $2.34 \mathrm{~g} / \mathrm{cm}^{3}$. The mass was added using tweezers and a more sophisticated procedure may lead to a higher value for the maximum mass allowed but the result obtained was consistent over several trials. A value of 73 $\mathrm{dyn} / \mathrm{cm}$ was used for $\gamma$, the surface tension of water, at the given ambient temperature. Also shown in Fig. 4 is the theoretical result, which itself has an uncertainty due to the uncertainties of the mass and dimensions of the disk. As is seen, there is good agreement between the experimental and 


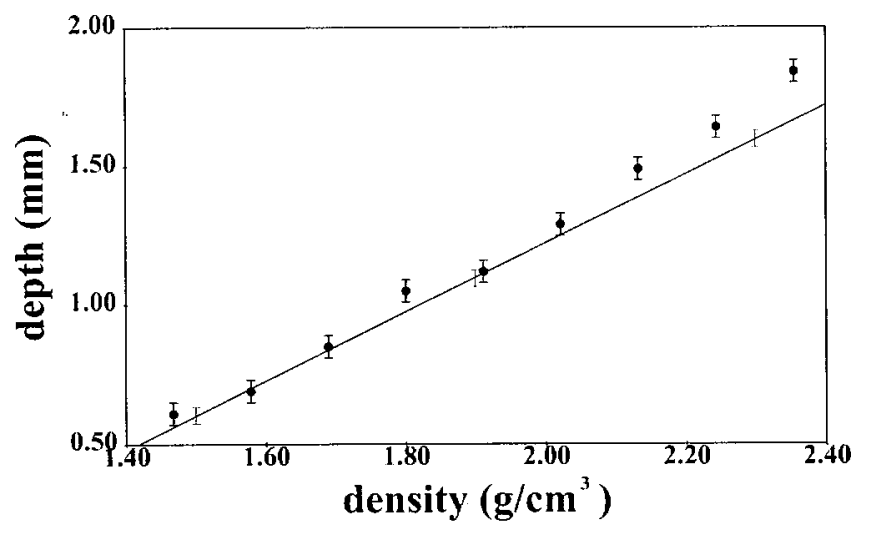

Fig. 4. Theoretical and experimental results for the acrylic disk for $\gamma$ $=73 \mathrm{dyn} / \mathrm{cm}$.

theoretical results up to depths, $y_{s}$, of $1.25 \mathrm{~mm}$. For larger depths up to a value of $1.84 \mathrm{~mm}$ when the disk broke through the surface, there appears to be a deviation from the theoretical result.

Figure 5 shows the result for an aluminum disk of radius $(9.66 \pm 0.03) \mathrm{mm}$ and thickness $(1.25 \pm 0.02) \mathrm{mm}$. Again measurements were made for every $100 \mathrm{mg}$ of mass added, corresponding in this case to an increase of $0.273 \mathrm{~g} / \mathrm{cm}^{3}$ in the equivalent density. In this case only $300 \mathrm{mg}$ of mass could be added until the disk broke through the surface at a total mass of $1.26 \mathrm{~g}$ or an equivalent density of $3.45 \mathrm{~g} / \mathrm{cm}^{3}$. Comparing the experimental result with the theoretical result a similar result to that of the acrylic disk was found. Initially good agreement is obtained, but as the mass was increased up to the point of maximum mass the experimental values give slightly larger depths than what is predicted by theory. Although the deviation between experiment and theory is relatively small, $10 \%$ difference for the worst case, the consistency of the deviation between the two disks for similar depths, $y_{s}$, would indicate it is a real feature. A lower value for $\gamma$, on the order of $60 \mathrm{dyn} / \mathrm{cm}$, would lead to good agreement between theory and experiment but as clean distilled water was used, there is no reason to expect such a low value of surface tension.

By using measured values of critical angles determined from various sensile drops placed on the disks the maximum

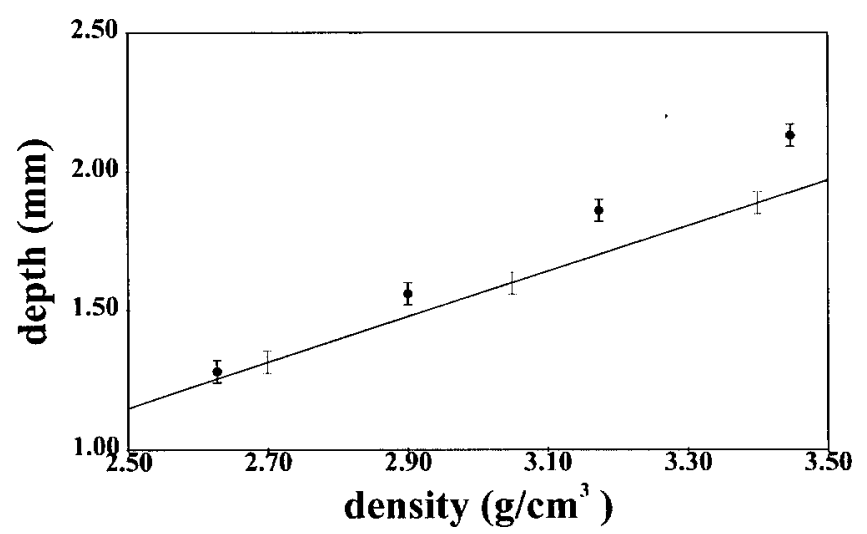

Fig. 5. Theoretical and experimental results for the aluminum disk for $\gamma$ $=73 \mathrm{dyn} / \mathrm{cm}$. theoretical mass that the two disks can reach can be determined. For the acrylic disk a value for the critical angle between water and acrylic of $42^{\circ} \pm 3^{\circ}$ was obtained. Using this value for $\theta_{0}$ results in a theoretical maximum allowed mass of $(2.20 \pm 0.10) \mathrm{g}$ for the acrylic disk. This corresponds well with the actual value of $2.12 \mathrm{~g}$ obtained from the experimental trials. In the case of the aluminum disk a value of $65^{\circ} \pm 3^{\circ}$ for the critical angle was obtained from the sensile drops. Using this value for $\theta_{0}$ results in a maximum allowed mass of $(1.54 \pm 0.05) \mathrm{g}$ for the aluminum disk. This is greater than the experimental maximum mass of $1.26 \mathrm{~g}$. The fact that the experimental values are lower is not surprising as the disks became quite unstable as the mass approached the critical point, the reason being that it was extremely difficult to keep the disk perfectly horizontal as mass was added to the disk. Also any contamination or irregularity at any point along the edge of the disk results in the water quickly moving in on the top face at that location.

\section{CONCLUSION}

Experimental determination of the depths two suspended disks fell beneath the water surface was compared with theoretical predictions. Good agreement was obtained for depths up to approximately $1.25 \mathrm{~mm}$, but for deeper depths discrepancies were found with experimental depths being up to $10 \%$ deeper than that predicted by theory. A lower value for the surface tension, $60 \mathrm{dyn} / \mathrm{cm}$, instead of $73 \mathrm{dyn} / \mathrm{cm}$ does lead to better agreement but, as already stated, the water was clean and since the discrepancies increase with depth, it is not expected that this is the explanation. A dependence of surface tension on the radius of curvature of the water surface would explain the deviation at deeper depths. Such an effect would be expected at the microscopic level, for radii of curvature of the surface on the scale of intermolecular distances, but the minimum radius of curvature for the above two disks, determined from the surface equation, is approximately $3 \mathrm{~mm}$. To determine the cause of the deviation, whether it be a physical effect or a problem with the experimental technique, the actual surface profile of the liquid should be obtained. This would be a difficult exercise as any measurements taken from the side would need to be corrected for distortions due to refractive effects.

The contribution made by the buoyant force was found to be dependent on both the size and density of the disk. The contribution increased with the size of the disk and decreased with increasing density. This result would be expected to hold in general for nondisk shapes as well. For an insect walking on water, the equivalent disk would have a radius $\ll 1 \mathrm{~mm}$ and an equivalent density $\gg 10 \mathrm{~g} / \mathrm{cm}^{3}$ and therefore it is legitimate for such problems to approximate the upward support force as being due solely to surface tension. However, for relatively larger objects and lower equivalent densities the buoyant force must be taken into account.

\footnotetext{
${ }^{1}$ A. V. Rapacchietta, A. W. Neumann, and S. N. Omenyi, "Force and Free-energy Analyses of Small Particles at Fluid Interfaces. I. Cylinders," J. Colloid Interface Sci. 59 (3), 541-554 (1977).

${ }^{2}$ A. V. Rapacchietta and A. W. Neumann, "Force and Free-energy Analyses of Small Particles at Fluid Interfaces. II. Spheres,' J. Colloid Interface Sci. 59 (3), 555-567 (1977)

${ }^{3}$ I. Bruce, 'CConcerning drops,' Am. J. Phys. 52 (12), 1102-1105 (1984). ${ }^{4}$ F. Behroozi et al., "The profile of a dew drop,' Am. J. Phys. 64 (9), 1120-1125 (1996).

${ }^{5}$ E. Kreyszig, Advanced Engineering Mathematics (Wiley, New York, 1972), 3rd ed., Chap. 18.
} 